\title{
FREQUÊNCIA DOS CASOS DE DENGUE NOS DISTRITOS DO MUNICÍPIO DE DUQUE DE CAXIAS, RIO DE JANEIRO, BRASIL
}

\author{
Karla PEDRO ${ }^{1}$, Renan DOCILE ${ }^{2}$, Edvaldo SILVA ${ }^{3,4}$, Tatiana DOCILE ${ }^{1,5,6,7} *$ \\ ${ }^{1}$ Faculdade de Filosofia, Ciências e Letras de Duque de Caxias, Departamento de Ciências Biológicas, Duque de \\ Caxias, Rio de Janeiro, Brasil. \\ ${ }^{2}$ Universidade do Estado do Rio de Janeiro, Instituto de Matemática e Estatística, Rio de Janeiro, Brasil. \\ ${ }^{3}$ Programa de Pós-Graduação em Educação profissional em Saúde, Escola Politécnica de Educação Profissional em \\ Saúde, Rio de Janeiro, Brasil. \\ ${ }^{4}$ Departamento de Vigilância em Saúde, Divisão de Informação em Saúde, Secretaria Municipal de Saúde, Duque de \\ Caxias, Rio de Janeiro, Brasil \\ ${ }^{5}$ Universidade Federal do Rio de Janeiro, Laboratório de Entomologia, Departamento de Zoologia, Rio de Janeiro, \\ Brasil. \\ ${ }^{6}$ Universidade Federal do Rio de Janeiro, Programa de Pós-Graduação em Ecologia, Rio de Janeiro, Brasil. \\ ${ }^{7}$ Centro Universitário Estadual da Zona Oeste, Laboratório de Biotecnologia Ambiental, Rio de Janeiro, Brasil. \\ *Autor para correspondência: tatidocile@gmail.com
}

http://dx.doi.org/10.18571/102

\section{RESUMO}

Dengue, Zika e Chikungunya são doenças transmitidas por vetores, cujo detectar os criadouros dos mosquitos torna-se vital para medidas de controle. $\mathrm{O}$ objetivo desse estudo foi avaliar os casos notificados de dengue no município de Duque de Caxias (Rio de Janeiro, Brasil) em seus quatro Distritos Sanitários, durante oito anos, compreendendo o período de 2007 a 2014. Essa região apresenta inúmeros problemas relacionados a processo de ocupação desordenada, densidade demográfica e altos índices de violência urbana e o município é organizado em quatro distritos. Os casos de dengue notificados à Secretaria Municipal de Saúde de Duque de Caxias e registrados na base municipal do SINAN (Sistema Nacional de Agravos de Notificação), referentes ao período de 2007 a 2014 foram utilizados neste estudo. Em 2008, 2011 e 2013, observou-se uma epidemia no município, que em 2008 tomou magnitude ainda maior e alcançou incidência de mais de mil casos a cada 100 mil habitantes. No período de 2009 e 2014, houve uma queda das incidências observadas, caracterizando-o como interepidêmico. Em relação aos casos de dengue em cada distrito, o primeiro e segundo distritos foram os que tiveram maior número de casos da doença, enquanto o quarto distrito foi o que teve menor número de casos em todos os anos estudados. Possivelmente, pelo fato do $1^{\circ}$ distrito (Centro) ter a maior parte das habitações e o $2^{\circ}$ distrito (Campos Elíseos), abrigar as indústrias. Enquanto o $3^{\circ}$ distrito (Imbariê) e $4^{\circ}$ distrito (Xerém) são considerados muito mais arborizados e com poucos núcleos habitacionais.

Palavras-chave: Saúde pública; Dengue; Aedes aegypti; Baixada Fluminense.

\begin{abstract}
Dengue cases frequency in the districts of the municipality of Duque de Caxias, Rio de Janeiro, Brazil. Dengue, Chikungunya and Zika are vector-borne diseases, which detect the breeding of mosquitoes becomes vital to control measures. The aim of this study was to evaluate reported cases of dengue in the city of Duque de Caxias (Rio de Janeiro, Brazil) in its four health districts, for eight years, covering the period 2007 to 2014. This region presents numerous problems related to disorderly process of occupation, population density and high rates of urban violence and the municipality is organized into four districts. Dengue cases reported to the Health Municipal of Duque de Caxias and registered in the municipal base SINAN (National System of Notifiable Diseases) for the period 2007-2014 were used in this study. In 2008, 2011
\end{abstract}


and 2013, there was an epidemic in the city, which in 2008 took even greater magnitude and incidence reached more than a thousand cases per 100 thousand inhabitants. Between 2009 and 2014, there was a decrease of the observed effects, characterizing it as interepidemic. For cases of dengue in each district, the first and second districts were those that had a higher number of cases of the disease, while the fourth district was what had fewer cases in all years studied. Possibly, because the 1st district (center) have most of the houses and the 2nd district (Campos Elísios), distribution industries. While the 3rd district (Imbariê) and 4th district (Xerém) are considered much more wooded and with few housing units.

Keywords: Public health; Dengue; Aedes aegypti; Baixada Fluminense.

\section{Introdução}

A crescente urbanização acelerada, poluição, degradação ambiental, deficiências de infraestrutura, saneamento e educação, todos esses fatores podem contribuir para o surgimento de doenças transmitidas por vetores que geram consequências indesejáveis para a qualidade de vida da população (FORATTINI, 1992).

Um dos grandes problemas das doenças transmitidas por vetores é conseguir a participação efetiva da população no seu controle. No caso específico da dengue, como a grande maioria dos criadouros dos mosquitos vetores encontram-se nas residências ou em suas imediações, esta questão torna-se vital para a eficácia das medidas de controle.

Sem vacinas e medicamentos específicos, as únicas respostas disponíveis ao desafio da dengue são o manejo clínico adequado, controle e prevenção um sistema de vigilância eficiente. A implementação destas estratégias é, entretanto, dificultada pela complexidade dos problemas sociais, como o pouco acesso e compreensão da informação pela população (KROEGER e NATHAN, 2006).

Dez arboviroses tem sido vista como importantes agentes de doenças no Brasil (FIGUEIREDO, 2007), o qual Aedes aegypti pode transmitir o vírus da dengue (DENV), o vírus da febre amarela (YFV), o vírus da Chikungunya (CHIKV) (FIGUEIREDO e FIGUEIREDO, 2014) e o vírus da Zika (ZIKV) (IOOS et al., 2014).

A dengue é uma doença infecciosa febril aguda, virose cujos vetores são mosquitos do gênero Aedes que transmitem a doença ao picar os indivíduos (VERONESI e FOCACCIA, 2005). Esse vírus é encontrado na forma de quatro sorotipos (DENV1, DENV2, DENV3 e DENV4), localizado na família flaviridaee gênero flavivirus. Alguns fatores sociais são responsáveis pela falência do controle na reprodução do Aedes aegypti, aumentando significativamente sua população e desses podemos citar: deficiência nos programas de controle do mosquito, êxodo rural, facilidade das migrações entre regiões e urbanização acelerada (CASALI et al., 2004).

A dengue é a principal doença reemergente da atualidade e quase $40 \%$ da população mundial vivem sob o risco de adquiri-la. $\mathrm{O}$ aumento do número de casos e a disseminação para novas áreas geográficas, especialmente as regiões tropicais, fazem da doença um importante problema de saúde pública (GUBLER, 2004; TAUIL, 2006). Reconhece-se que, assim como em outras viroses reemergentes, seu desencadeamento se relaciona às atividades humanas que modificam o ambiente (FARRAR et al., 2007). A influência das desigualdades sociais sobre a situação de saúde de populações vem sendo discutida em diversos estudos de forma ampla (TEIXEIRA e PUNGIRUM, 2005) ou sob análises de eventos específicos (CHIESA, 2002; ISHITANI, 2006). Alguns autores abordam a relação entre variáveis relacionadas às condições de vida da população e a ocorrência da dengue; entretanto os resultados encontrados são divergentes (COSTA e NATAL, 1998; MARZOCHI, 2004). A maioria destes estudos utilizou metodologia em que as variáveis relativas às condições de vida foram abordadas num mesmo 
nível, não contemplando o contexto social enquanto unidade complexa, no qual fatores socioculturais e estruturais urbanos geram uma realidade única em cada local, favorecendo ou não a disseminação da dengue.

No Brasil, a extensa disseminação do dengue ocorreu em 1986 no Rio de Janeiro, espalhando-se para vários estados (LLOYD, 1994; DONALISIO, 1999). Em 2008, conforme informação do Portal da Saúde do RJ - Tabnet, dentre os casos notificados, foram confirmados 102.250 casos de dengue em residentes em todo o estado do Rio de Janeiro. O município do Rio de Janeiro apresentou o maior número de casos confirmados em residentes (32.301), seguido pelo município de Duque de Caxias, com 15.219 casos confirmados em residentes. O município de Duque de Caxias faz parte da Região Metropolitana I do estado do Rio de Janeiro, juntamente com Belford Roxo, Itaguaí, Japeri, Mesquita, Nilópolis, Nova Iguaçu, Magé, Queimados, Seropédica, São João de Meriti e Rio de Janeiro. Duque de Caxias ocupa 35\% da Baixada Fluminense e segundo dados apresentados pelo IBGE para o censo de 2010, constitui-se como o município mais populoso da Baixada Fluminense, com 855.048 habitantes. Apesar de ser um município com muitos casos da doença, ainda são poucos os estudos sobre essa grande área afetada. Tendo em vista os frequentes casos da doença, o objetivo desse estudo foi avaliar, analisar e divulgar a frequência dos casos de dengue notificados em residentes no município de Duque de Caxias, Rio de Janeiro- Brasil com diagnóstico entre os anos 2007 a 2014 e distribuídos conforme área distrital.

\section{Metodologia}

O município Duque de Caxias é atualmente, o terceiro município mais populoso do Estado do Rio de Janeiro, com 665.343 habitantes recenseados pelo IBGE em 1991. Possui uma área de $462,6 \mathrm{~km}^{2}$ e sua densidade demográfica é, portanto de $1.438,27 \mathrm{hab} . / \mathrm{km}^{2}$. É uma região que apresenta inúmeros problemas relacionados a processo de ocupação desordenada, densidade demográfica e altos índices de violência urbana (Brasil, 2005). O município é organizado em quatro distritos: $1^{\mathrm{o}}$ distrito (Centro), onde a maior parte das habitações e as atividades econômicas se concentram; $2^{\circ}$ distrito (Campos Elíseos), no qual se localizam as indústrias de transformação e distribuição de produtos manufaturados; $3^{\circ}$ distrito (Imbariê), área de grandes reservas florestais e estabelecimentos não ocupados e $4^{\circ}$ distrito (Xerém) considerado rural, com poucos núcleos habitacionais (Duque de Caxias, 2002) (Figura 1).

Os casos de dengue notificados à Secretaria Municipal de Saúde de Duque de Caxias e registrados no SINAN (Sistema Nacional de Agravos de Notificação), referentes ao período de 2007 a 2014 foram fornecidos pela prefeitura e utilizados neste estudo.

Neste trabalho ecológico de base censitária, utilizaram-se os quatro distritos sanitários como unidade mínima de análise, uma vez que não foi possível agrupar os registros por setores censitários com base na recuperação dos endereços dos pacientes notificados sobre o eixo de ruas do município. Os anos estudados foram agrupados em períodos epidêmicos e interepidêmicos para a análise espaço-temporal, utilizando-se a incidência acumulada como medida de análise da ocorrência de dengue.

As análises dos dados foram realizadas na Faculdade de Filosofia, Ciências e Letras de Duque de Caxias - FEUDUC localizada no bairro de Gramacho, no município de Duque de Caxias, Rio de Janeiro, Brasil. 


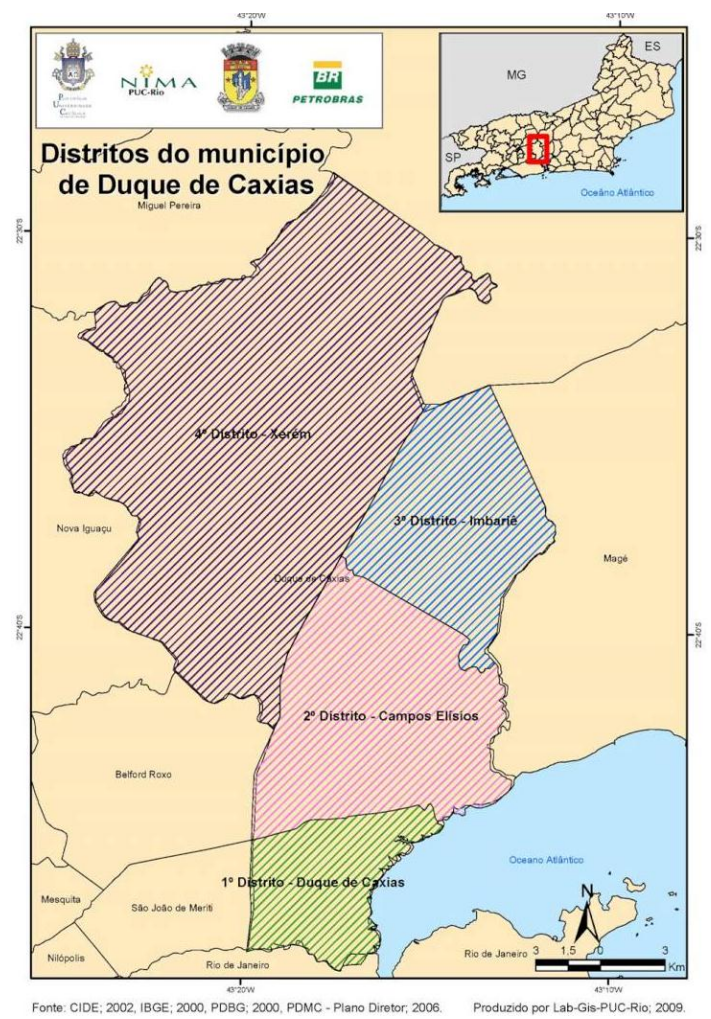

Figura 1: Mapa com a separação dos quatro distritos de Duque de Caxias e acima a localização do município no Estado do Rio de Janeiro. Fonte: IBGE (2016).

\section{Resultados e discussão}

Nos anos estudados, caracterizam-se quatro períodos distintos segundo a incidência da dengue. Nos anos de 2009 e 2014, as taxas encontradas foram menores que as de outros períodos. Em 2008, 2011 e 2013, observou-se uma epidemia no município analisado, que em 2008 tomou magnitude ainda maior e alcançou incidência de mais de mil casos a cada 100 mil habitantes. No período de 2009 e 2014, houve uma queda das incidências observadas, caracterizando-o como interepidêmico.

Em relação aos casos de dengue em cada distrito, o $1^{\circ}$ e o $2^{\circ}$ distritos (Duque de Caxias e Campos Elíseos, respectivamente) foram os que apresentaram maiores números de casos notificados da doença, possivelmente, pelo fato de o $1^{\circ}$ distrito concentrar a maior parte da população do município e o $2^{\circ}$ distrito abrigar as indústrias de transformação e distribuição de produtos manufaturados, enquanto o $3^{\circ}$ e o $4^{\circ}$ distritos (Imbariê e Xerém, respectivamente) são considerados muito mais arborizados e inferior número de núcleos habitacionais. (Duque de Caxias, 2002). O $4^{\circ}$ distrito (Xerém) foi o que apresentou menor número de casos notificados em todos os anos estudados.

O primeiro distrito é o que possui o maior número de casos na maioria dos anos, o segundo distrito vem em segundo lugar. No segundo ano (2008) de análise os números de casos aumentaram muito, no ano seguinte (2009) houve uma redução considerável, nos 2 anos seguintes os números de casos voltaram a aumentar. Em 2012 houve uma queda no número de casos, em 2013 houve aumento e em 2014, último ano de análise, os números de casos diminuíram novamente, ou seja, como mostram na tabela e figuras 2 e 3 , houve com o passar dos anos picos de incidência de dengue, mas não podemos afirmar que com o passar dos anos aumentaram os números de casos diagnosticados da doença. Houve um intervalo entre altos e baixos números de casos da doença. Na figura 2 é possível verificar três anos em que a doença 
alcança um alto pico: 20082011 e 2013. Uma das epidemias de dengue que ocorreu no estado do Rio de Janeiro em 2008 causada pelos vírus tipo 2 (principalmente) e 3, segundo dados da Secretaria de Estado de Saúde em 2008. Entre os critérios considerados para que um município entre em epidemia está o registro de mais de 300 casos por 100 mil habitantes, curva ascendente de transmissão da doença sustentada por três semanas ou mais consecutivas, e com números acima do limite esperado para a localidade num determinado período de tempo (Secretaria de Saúde 2008).

Tabela 1: Total do número de casos de dengue por distrito do Município de Duque de Caxias- RJ, referentes ao período de 2007 a 2014 fornecidos pela Secretaria Municipal de Saúde de Duque de Caxias.

\begin{tabular}{lllllllll}
\hline Distritos & 2007 & 2008 & 2009 & 2010 & 2011 & 2012 & 2013 & 2014 \\
$\begin{array}{l}1^{\circ} \text { DISTRITO } \\
\text { D. CAXIAS }\end{array}$ & 132 & 5063 & 33 & 235 & 3377 & 1.692 & 2849 & 97 \\
$\begin{array}{l}2^{\circ} \text { DISTRITO } \\
\text { C. ELÍSEOS }\end{array}$ & 121 & 4690 & 28 & 284 & 3505 & 715 & 2073 & 125 \\
$\begin{array}{l}1^{\circ} \text { DISTRITO } \\
\text { IMBARIE }\end{array}$ & 159 & 4251 & 59 & 759 & 1874 & 196 & 1607 & 16 \\
$\begin{array}{l}4^{\circ} \text { DISTRITO } \\
\text { XERÉM }\end{array}$ & 13 & 711 & 02 & 32 & 357 & 110 & 353 & 06 \\
\hline TOTAL & 425 & 14.715 & 122 & 1.310 & 9.113 & 2.713 & 6.882 & 244 \\
\hline
\end{tabular}

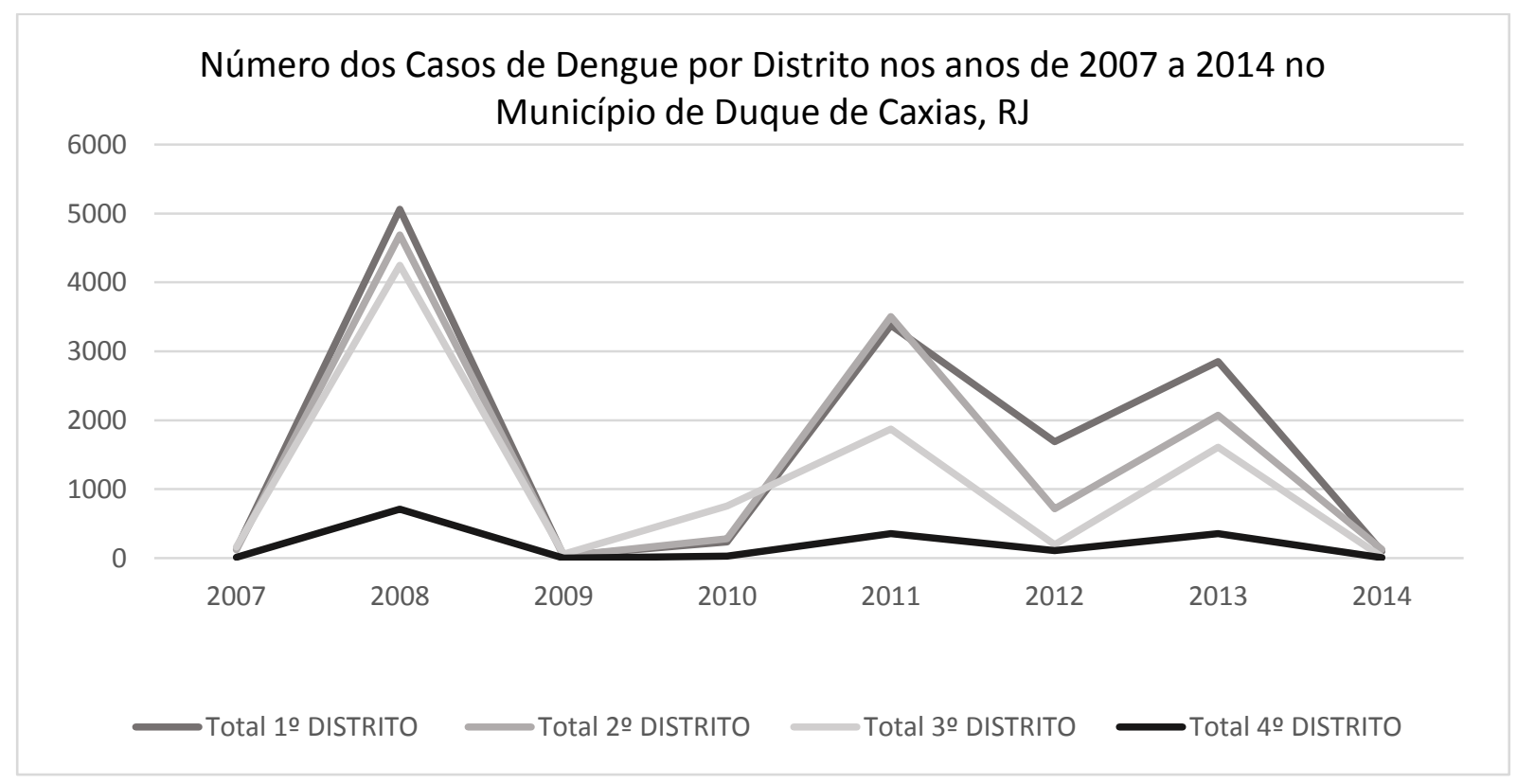

Figura 2: Gráfico temporal elaborado ilustrando os números de casos de dengue por distrito do Município de Duque de Caxias- RJ, referentes ao período de 2007 a 2014. 
Distribuição Proporcional dos Casos de Dengue por Distrito nos Anos de 2007 a 2014 no Município de Duque de Caxias, RJ

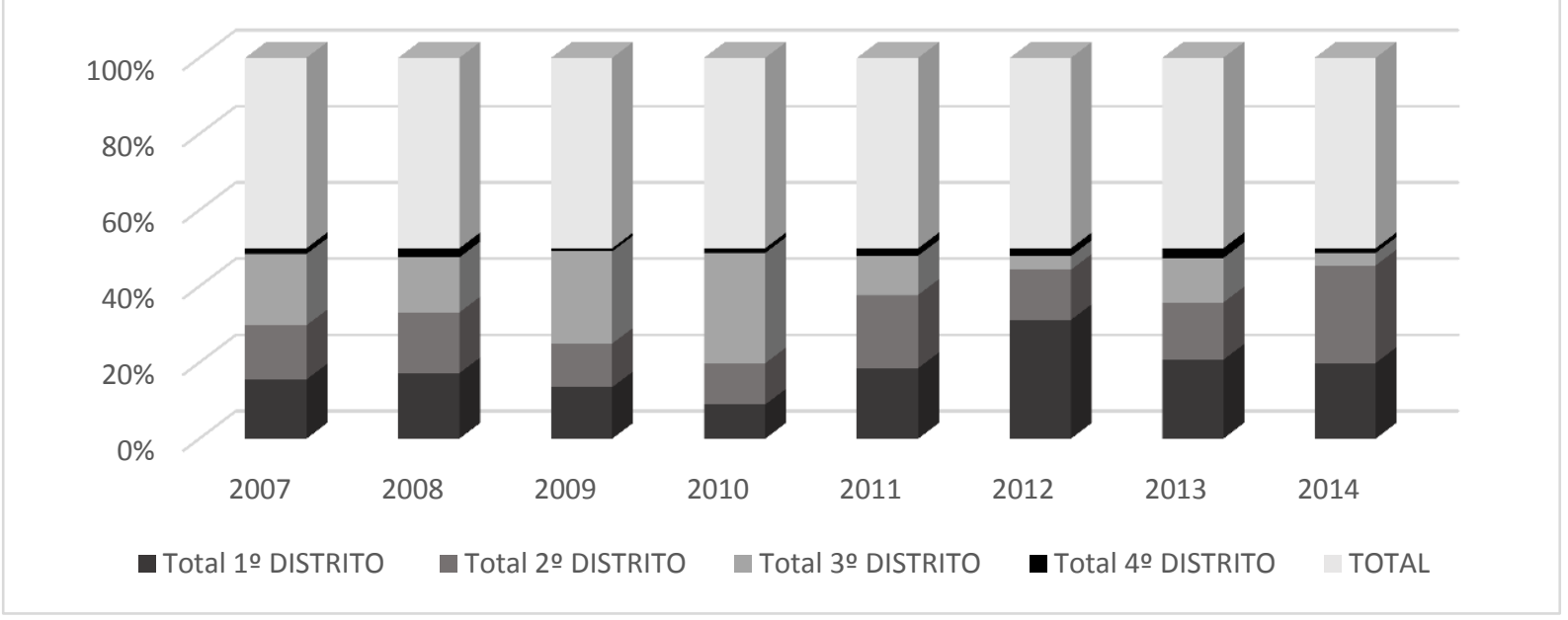

Figura 3: Gráfico em barra, elaborado ilustrando a distribuição proporcional dos casos de dengue por distrito do Município de Duque de Caxias- RJ, referentes ao período de 2007 a 2014.

O município de Duque de Caxias está a cerca de quinze minutos do Aeroporto Internacional Tom Jobim e da Universidade Federal do Rio de Janeiro - UFRJ. Faz parte da região metropolitana e, apesar da pequena distância em relação à cidade do Rio de Janeiro, apresenta índices de pobreza extrema. Sua população corresponde a $5,4 \%$ da população do Estado. Considerando o município como um todo, a população que vive abaixo da linha de pobreza extrema está em torno de $14 \%$, quase o dobro da média do estado do Rio de Janeiro. Dentro do próprio município há áreas críticas de pobreza, atingindo até cerca de $20 \%$ dos habitantes, conforme apontam alguns indicadores alguns indicadores sociais de Duque de Caxias: cerca de $20 \%$ da população não são alfabetizados; $58 \%$ dos domicílios apresentam infraestrutura inadequada; os trabalhadores com vínculo formal estão em torno de apenas $25 \%$; aproximadamente $50 \%$ recebem rendimentos acima de três salários mínimos; a população se distribui em estratos etários variados (SALLES-COSTA, 2007).

Segundo Leontisini et al. (1993), a participação efetiva da comunidade no controle apresenta, além do incentivo à comunidade para resolução de seus problemas, uma vantagem econômica, porque parte dos seus custos são assumidos pela própria comunidade. Na realidade atual, onde impera uma falta constante de verbas para o setor de saúde, isto pode ser um diferencial importante.

É importante ressaltar a necessidade de melhorar o controle vetorial nos municípios infestados com Aedes aegypti, já que somente essa espécie no Brasil está, até o momento, associada à transmissão de três arboviroses, dengue, Chikungunya e Zika e, também, o enorme desafio da vigilância epidemiológica em reconhecer precocemente as novas áreas com transmissão para minimizar o impacto dessas doenças na população.

Atualmente, ainda se busca a descoberta de uma vacina, mas a grande dificuldade é a imunização contra os quatro tipos de vírus da dengue (FIGUEIREDO, 1999). Assim, a única forma de prevenção existente consiste no conhecimento, estudo e controle do mosquito transmissor (DOCILE, 2012).

Leontisini et al. (1993) dão outras sugestões importantes a respeito de como aumentar o impacto das atividades de participação comunitária sobre os índices de infestação do Aedes aegypti. Para eles, deveriam ser incorporadas atividades de reciclagem de resíduos, desenvolvimento de atividades participativas nos locais de trabalho, integração de atividades 
comunitárias com a de rotina dos serviços governamentais, etc. O aprofundamento dessa discussão com certeza poderá gerar mudanças nos trabalhos educativos desenvolvidos, no sentido de se conseguirem melhores resultados.

\section{Conclusão}

Existe um intervalo entre altos e baixos números de casos da doença, representando alguns anos epidêmicos. O primeiro e segundo distritos são mais povoados e apresentam mais números de casos da doença. Enquanto, o terceiro e quarto distrito são muito mais arborizados e com poucos núcleos habitacionais apresentando menor número de casos em todos os anos estudados. A diferença social e geográfica dos distritos influência no resultado do número de casos.

\section{Agradecimentos}

Os autores da pesquisa agradecem à Faculdade de Filosofia, Ciências e Letras de Duque de Caxias (FEUDUC) e à Secretaria de Saúde do Município de Duque de Caxias pelo apoio. Agradecem também à Brenda Cavalcante pela contribuição da revisão da parte escrita.

\section{Referências}

CASALI, C. G. A epidemia de dengue/dengue hemorrágico no município do Rio de Janeiro, 2001/2002. Revista da Sociedade Brasileira de Medicina Tropical, Uberaba, v. 37, n. 4, 2004.

CHIESA, A. M.; WESTPHAL, M. F.; KASHIWAGI, N. M. Geoprocessamento e a promoção da saúde: desigualdades sociais e ambientais em São Paulo. Revista de Saúde Pública v. 36, p. 559-567, 2002.

COSTA, A. I. P.; NATAL, D. Distribuição espacial da dengue e determinantes socioeconômicos em localidade urbana no Sudeste do Brasil. Revista de Saúde Pública, v. 32, p. 232-6, 1998.

DOCILE, T. N. Um olhar ecológico sobre os vetores do Dengue. In: FIGUEIRÓ, R. Saúde \& Ambiente: Da Educação Ambiental à Ecologia de Doenças, 1. ed. Rio de Janeiro: UNIFOA, Volta Redonda, 2012. p. 24-31.

DONALISIO, M. R. O dengue no espaço habitado. Editora Hucitec, São Paulo, 1999.

FARRAR, J.; FOCKS, D.; GUBLER, D.; BARRERA, R.; GUZMAN, M. G.; SIMMONS, C. Towards a global dengue research agenda. Tropical Med. Int. Health, v. 12, p. 695-699, 2007.

FIGUEIREDO, L. T. Emergent arboviruses in Brazil. Revista da Sociedade Brasileira de Medicina Tropical, v. 40, p. 224-229, 2007

FIGUEIREDO, L. T. M. Patogenia das infecções pelos vírus do dengue. Medicina, v. 32, p. 1520,1999 .

FIGUEIREDO, M. L.; FIGUEIREDO, L. T. Emerging alpha viruses in the Americas: Chikungunya and Mayaro. Revista da Sociedade Brasileira de Medicina Tropical, v. 47, p. 677-683, 2014. 
FORATTINI, O. P. Ecologia, epidemiologia e sociedade. São Paulo: Artes Médicas/Edusp, 1992.

GUBLER, D. J. The changing epidemiology of yellow fever and dengue, 1900 to 2003: full circle? Comp Immunol Microbiol Infect Dis, v. 27, p. 319-330, 2004.

INSTITUTO BRASILEIRO DE GEOGRAFIA E ESTATÍSTICA- IBGE (Brasil). Censo sobre os casos de dengue no Rio de Janeiro. Disponível em: <http://www.censo2010.ibge.gov.br/sinopse/index.php?uf=33\&dados=29>. Acesso em 7 abril, 2016.

INSTITUTO BRASILEIRO DE GEOGRAFIA E ESTATÍSTICA- IBGE. Censo Demográfico. Disponível em: <https://www.censo1990.ibge.gov.br>. Acesso em 5 abril, 2016.

IOOS, S.; MALLET, H. P.; GOFFART, I. L.; GAUTHIER, V.; CARDOSO, T.; HERIDA, M. Current Zika vírus epidemiology and recent epidemics. Med Mal Infect, v. 44, p. 302-307, 2014.

ISHITANI, L. H.; FRANCO, G. C.; PERPÉTUO, I. H. O.; FRANÇA, E. Desigualdade social e mortalidade precoce por doenças cardiovasculares no Brasil. Revista de Saúde Pública, v. 40, p. 684-691, 2006.

KROEGER, A.; NATHAN, M. B. Dengue: setting the global research agenda. The Lancet, USA, v. 368, p.2193-2195, dez. 2006.

LEONTISINI, E.; GIL, E.; KENDALL, C.; CLARK, G. G. Effect of a community-based Aedesaegypti control programme on mosquito larval production sites in El Progresso, Honduras. Transactions of the Royal Society of Tropical Medicine and Hygiene, v. 87, p. 267-271, 1993.

LLOYD, L.S.; WINCH, P.; ORTEGA-CANTO, J.; KENDALL, C. The design of a communitybased health education intervention for the control of Aedes aegypti. American Journal of Tropical Medicine and Hygiene, v. 50, p. 401-411, 1994.

MARZOCHI, K. B. F. Dengue endêmico: o desafio das estratégias de vigilância. Revista da Sociedade Brasileira de Medicina Tropical, v. 37, p. 413-5, 2004.

SALLES-COSTA, R. Avaliação alimentar em duque de Caxias (RJ). Estudos avançados, v. 21 (60), 2007.

SECRETARIA DE ESTADO DE SAÚDE DO RIO DE JANEIRO. Disponível em: < http://sistemas.saude.rj.gov.br/tabnet/deftohtm.exe?sinan/denguef.def $>$. Acessado em 7 abril, 2016.

SECRETARIA DE SAÚDE 2008. Disponível <http://www.combateadengue.com.br/relatorio-de-casos-de-dengue-no-rio-de-janeiro>. Acessado em 7 abril, 2016.

TAUIL, P. L. Perspectivas de controle de doenças transmitidas por vetores no Brasil. Revista da Sociedade Brasileira de Medicina Tropical, v. 39, p. 275-277, 2006. 
TEIXEIRA, J. C.; PUNGIRUM, M. E. M. C. Análise da associação entre saneamento e saúde nos países da América Latina e do Caribe, empregando dados secundários do banco de dados da Organização Pan-Americana da Saúde - OPAS. Revista Brasileira de Epidemiologia, v. 8, p. 365-376, 2005.

VERONESI, R.; FOCACCIA, R. Tratado de infectologia. $3^{\mathrm{a}}$. ed. São Paulo: Atheneu, 2005. 